\title{
VITAMIN A IN BOVINE SPERM ACROSOMES
}

\author{
K. K. GAMBHIR AND B. S. AHLUWALIA* \\ Endocrine Research Laboratory, Departments of Medicine and \\ Obstetrics and Gynecology, Howard University Medical School, \\ Washington, D.C. 20059, U.S.A.
}

(Received 29th August 1974)

\begin{abstract}
Summary. Bull semen was collected artificially and vitamin A was determined in the intact spermatozoa, the acrosomes and the sperm tails. The results show that over $90 \%$ of the total vitamin $A$ in the spermatozoa was present in the acrosomes and that the sperm tails were completely devoid of vitamin A. The concentration of vitamin A varied from 224 to $364 \mathrm{ng} / 10^{9}$ spermatozoa.
\end{abstract}

Vitamin A has a direct localized effect on the normal differentiation of the testicular germinal epithelium (Palludan, 1966; Ahluwalia \& Bieri, 1970, 1971). Using labelled vitamin A, we have shown that the Sertoli cell is the principal site of incorporation of radioactivity (B. S. Ahluwalia, K. K. Gambhir and H. S. Sekhon, unpublished data). Since vitamin A may be one of the 'nutrients' that developing spermatozoa receive from the Sertoli cells, it seemed of interest to determine whether spermatozoa contain vitamin $A$ and, if so, in which component the vitamin is concentrated. Because it can be collected conveniently and in large volumes, we chose bull semen for this study.

Five pure bred Holstein bulls of identical age and breeding records were used. The semen collection schedule was kept uniform throughout the study. Three ejaculates were collected with an artificial vagina each time. The first ejaculate was discarded because the quality of the semen from the first ejaculate is always poor in bulls on a once per week semen collection schedule. The remaining two ejaculates were pooled. An aliquot was used to determine the number of spermatozoa/ml semen from each sample, and the remainder was packed in dry ice and brought to the laboratory for analysis.

Vitamin A (retinyl-11,12-[ $\left.{ }^{3} \mathrm{H}_{2}\right]$ acetate-sp. act. $252 \mu \mathrm{Ci} / \mathrm{mg}$ ) was a gift from Hoffman-La Roche. All solvents were of spectroquality and were purchased from Matheson Coleman and Bell Manufacturing Chemists.

The semen was thawed and $10.0 \mathrm{ml}$ were centrifuged at $4000 \mathrm{~g}$ for $20 \mathrm{~min}$ at $4^{\circ} \mathrm{C}$. The seminal plasma was discarded and the pellet containing spermatozoa was washed twice with $5 \mathrm{ml}$ of $0.9 \%$ saline at room temperature. The contents were centrifuged at $4000 \mathrm{~g}$ for $10 \mathrm{~min}$ each time. The washed spermatozoa were suspended in $2.0 \mathrm{ml}$ physiological saline and an aliquot was used to observe microscopically any structural damage to the spermatozoa. No obvious damage to the spermatozoa was detected. The suspension containing spermatozoa was

\footnotetext{
* Reprint requests to Dr Ahluwalia.
} 
then used for acrosomal extraction (Fraction 1) according to the procedure of Bernstein \& Teichman (1973). The aqueous acrosomal suspension was used for vitamin A extraction. The residue containing spermatozoa partly depleted of acrosomes (Fraction 2) was also used for vitamin A determination because the efficiency of the acrosomal detachment procedure does not exceed 70 to $80 \%$. After suspending the residue in $2.0 \mathrm{ml}$ of $0.9 \%$ saline, the material was subjected to sonication in a Raytheon sonicator for $5 \mathrm{~min}$ with the controls set at the maximum to ensure complete disruption of the membranes. The sonicated material was used for determination of vitamin A content.

In the study which involved the determination of vitamin $A$ in sperm heads, $10 \mathrm{ml}$ semen was first thawed and the spermatozoa were separated from the seminal plasma as described above. The pellet containing the spermatozoa was washed twice with normal saline and the washed residue was resuspended in $5 \mathrm{ml}$ of $0.9 \%$ saline. The suspension containing the spermatozoa was then subjected to sonic oscillation for $3 \frac{1}{2} \mathrm{~min}$ with the controls set at $0.5 \mathrm{~A}$. The optimum time and control settings in the sonic oscillator were determined in a series of preliminary studies to ensure complete disruption of the sperm heads from the tails. Approximately 90 to $95 \%$ of the spermatozoa were found to be completely disrupted by this method when examined under the microscope. The fractions containing the sperm heads (Fraction 3) and tails (Fraction 4) were then separated according to our procedure described elsewhere (Ahluwalia \& Holman, 1969). Fractions 3 and 4 were washed with normal saline before carrying out the vitamin A estimation procedure.

Fractions 1, 2 and 4 and intact washed spermatozoa were used for vitamin $\mathrm{A}$ determination. Fraction 4 and intact washed spermatozoa were subjected to extensive sonication before vitamin A extraction as already described for the preparation of Fraction 2. Fraction 3 was not used for analysis because of the possibility of damage to the acrosomal membranes during sonication allowing vitamin A to escape into the surrounding fluid. A trace amount of labelled vitamin A acetate was added to each fraction before the extraction procedure to estimate recovery. The extraction procedure was carried out in two steps. In step 1, each fraction was mixed with an equal volume of ethyl alcohol and the contents were shaken vigorously for $5 \mathrm{~min}$. The contents were then extracted twice, each time with $10 \mathrm{ml}$ hexane, and the extract was collected. In step 2, the residue left after extraction with hexane was subjected to digestion after adding $1.0 \mathrm{ml}$ of $10 \%$ alcoholic $\mathrm{KOH}$ and $0.5 \mathrm{ml}$ of $1 \%$ pyrogallol as an antioxidant. The mixture was cooled and extracted with three $5.0-\mathrm{ml}$ vols of hexane. The extracts obtained in both steps were pooled and dried under nitrogen. The efficiency of the procedure, as determined by the amount of radioactivity in an aliquot at the end of the extraction procedure, was 75 to $80 \%$. The vitamin A content was determined fluorometrically (Aminco Bowman) using $330 \mathrm{~nm}$ and $470 \mathrm{~nm}$ as excitation and emission wavelengths according to the method described by Thompson, Erdody, Brien \& Murray (1971).

The results (Table 1 ) indicated that approximately $70 \%$ of the total vitamin A present in the spermatozoa was in Fraction 1 and that Fraction 2 contained approximately $27 \%$. Since the efficiency of the procedure used for acrosomal detachment in the study did not exceed 70 to $80 \%$ (Bernstein \& Teichman, 
1973), it was expected that the spermatozoa in Fraction 2 would contain vitamin A. Together Fractions 1 and 2 contained almost all the vitamin A present in the spermatozoa. From these results, it was concluded that Fraction 1 was the principal site of the localization of vitamin A in the spermatozoon. From preliminary data, the vitamin A appeared to be present in esterified form.

The presence of vitamin A in Fraction 1 and its link to physiological function is highly speculative. Acrosomal material is a mucopolysaccharide made up of units containing galactose, mannose, fucose and hexosamine, and this mucopolysaccharide forms a complex with protein and lipids (Berry \& Mayer, 1959). The possibility that vitamin $A$ is associated with a mucopolysaccharide-proteinlipid complex is under investigation. The rôle of vitamin A in mucopolysaccharide biosynthesis is well known (Wolf \& Johnson, 1960); therefore, the possibility that vitamin $\mathrm{A}$ is associated with an acrosomal enzyme, hyaluronidase, which brings about the depolymerization and hydrolysis of hyaluronic acid, is an attractive hypothesis.

Table 1. Vitamin A content of bovine spermatozoa and sperm components

\begin{tabular}{lcc}
\hline \multicolumn{1}{c}{ Fraction } & $\begin{array}{c}\text { No. of } \\
\text { samples }\end{array}$ & $\begin{array}{c}\text { Vitamin } A \\
\text { (ng/109 }\end{array}$ \\
\hline Intact spermatozoa)
\end{tabular}

Values expressed as means \pm S.D. Fraction 3 , containing the sperm heads, was not used for analysis-see text.

* Not detectable.

The means by which vitamin $\mathrm{A}$ is incorporated into the acrosomes need clarification. Our study (B. S. Ahluwalia, K. K. Gambhir and H. S. Sekhon, unpublished data) showing that the Sertoli cell is the major site of incorporation of labelled vitamin A suggests that the vitamin is probably incorporated into the acrosomes of the spermatozoa during their development. The spermatozoa of several species, including man, have been reported to contain metallic ions; among these, zinc appears to be predominant (Mann, 1964). Whether there is a functional association between zinc and vitamin $A$ in the spermatozoa is not known, but our preliminary study showing an enhancement in the uptake of labelled vitamin A by isolated seminiferous tubules in the presence of zinc is of interest (Ahluwalia, Devi \& Clark, 1973). The absence of vitamin A in Fraction 4 does not preclude the possibility of a vitamin A link with the physiological function of the sperm tails; it is more likely, however, that vitamin A is not involved in sperm movement.

This research was supported in part by National Institutes of Health Grant HD06045-02. We thank North American Breeders, Inc., Berryville, Virginia, for their generous supply of bull semen. 


\section{REFERENCES}

Ahluwalia, B. \& Bieri, J. (1970) Effects of exogenous hormones on the male reproductive organs of vitamin A deficient rats. $\mathcal{F}$. Nutr. 100, 715-724.

Ahluwalia, B. \& Bieri, J. (1971) Local stimulatory effect of vitamin A on spermatogenesis in the rat. F. Nutr. 101, 141-152.

Ahluwalia, B., DeVI, A. \& Clark, F. J. (1973) Enhancement with zinc in the incorporation of labelled vitamin A acetate in the seminiferous tubules of rat testes. Fedn Proc. Fedn Am. Socs exp. Biol. 32, 4093.

Ahluwalia, B. \& Holman, R. T. (1969) Fatty acid composition of lipids of bull, boar, rabbit and human semen. 7. Reprod. Fert. 18, 431-437.

Bernstein, M. H. \& Teichman, R. J. (1973) A chemical procedure for extraction of the acrosomes of mammalian spermatozoa. 7. Reprod. Fert. 33, 239-244.

BERRY, R. E. \& MAYER, D. T. (1959) The isolation of some cellular constituents of bovine spermatozoa. Expl Cell Res. 18, 398-401.

Mann, T. (1964) The Biochemistry of Semen and of the Male Reproductive Tract, p. 126. Wiley, New York.

Palludan, B. (1966) Direct effect of vitamin A on boar testis. Nature, Lond. 211, 639-640.

Thompson, J. N., Erdody, P., Brien, R. \& MurRay, T. K. (1971) Fluorometric determination of vitamin $\mathrm{A}$ in human blood and liver. Biochem. Med. 5, 67-89.

Wolf, G. \& Johnson, D. C. (1960) Vitamin A and mucopolysaccharide biosynthesis. Vitams Horm. 18, $439-483$. 